\title{
Subcarrier and Power Allocation for the Downlink of Multiuser OFDM Transmission
}

\author{
KHALID EL BAAMRANI ${ }^{1}$, ABDELLAH AIT OUAHMAN ${ }^{1}$, VICTOR P. GIL JIMÉNEZ ${ }^{2}$, \\ ANA GARCIA ARMADA ${ }^{2}$ and SAID ALLAKI ${ }^{3}$ \\ ${ }^{1}$ Department of Physique, University Cadi Ayyad Marrakech, Morocco \\ E-mails: elbaamrani@ucam.ac.ma; ouahman@ucam.ac.ma \\ ${ }^{2}$ Department of Signal Theory and Communications, University Carlos III Madrid, Spain \\ E-mails: vjimenez@tsc.uc3m.es; agarcia@tsc.uc3m.es \\ ${ }^{3}$ National Institute of Posts and Telecommunications, Rabat, Morocco \\ E-mail:allaki@inpt.ac.ma
}

\begin{abstract}
In this paper, a new algorithm for subcarrier and power allocation for the downlink of multiuser OFDM transmission is presented. The proposed algorithm is more stable and it offers a lower complexity and better performance than previous existing algorithms.
\end{abstract}

Keywords: OFDM, multiuser, subcarriers allocation

\section{Introduction}

The main difficult in achieving high data rates on the wireless channel is known to be the time dispersive charateristic of channels due to the existence of multiple paths, which have different time delays and attenuations. Due to its high spectrum efficien y and high ability to combat multipath fading problems, Orthogonal Frequency Division Multiplexing (OFDM) modulation is considered a promising technique for achieving the high bit rates required for wireless multimedia services over the time dispersive multipath channel. Multicarrier modulation systems such as OFDM can assign different modulation sizes to each subcarrier according to the signal to noise ratio (SNR) on that subcarrier [1], a scheme known as bit loading.

Adaptive bit-loading can be used to improve the performance of multicarrier systems by allocating more bits per symbol in subcarriers with higher signal to noise ratio. In this way, different bits and power can be allocated to each subcarrier depending on channel quality.

Several different optimization techniques have been proposed in multiuser OFDM literature for adaptive modulation. They can be classifie in two types: Margin Adaptive (MA) [2-5] and Rate Adaptive (RA) [6,7]. The margin adaptive objective is to achieve the minimum overall transmit power given the constraints on the users data rate and Bit Error Rate (BER). The rate adaptive objective is to maximize each user data rate with a total transmit power constraint. Wong et al. proposed an iterative searching algoithm [2] known as multiuser adaptive OFDM, which applies Lagrangian relaxation to solve the MA optimization. They have also formulated the optimization problem of combined subcarrier, bit and power allocation and obtained a tight lower bound of overall transmit power. It is a computaionally extensive algorithm, requiring a large number of iterations to converge, due to he nature of its nonlinear optimization algorithm. 
In [3], the nonlinear optimisation problems were transformed into a linear optimization problem with integer variables. The optimal solution can be achieved by integer programming. However, by using integer programming, the complexity increases exponentially with the number of constraints and variables. Didem Kivanc and Hui Liu [4] proposed the famous Graving Greedy subcarrier and power allocation algorithm. The algorithm was separated into two stages: In the firs step, the number of subcarriers that each user will get is determined based on the users' average signal-to-noise ratio (BABS algorithm). In the second stage of the algorithm, it find the best assignment of subcarriers to users. Two different approaches are presented, the Rate-Craving Greedy (RCG) algorithm begins with an estimate of the users' transmission rate on each channel and aims to maximize the total transmission rate and the Amplitude-Craving Greedy (ACG) algorithm is a modificatio of RCG which achieves comparable performance at reduced computational complexity. In [5], after analysis of the ACG algorithm, Li proposed a modifie ACG algorithm to further improve the system performance.

In this paper, a margin adaptive optimization is proposed for multiuser downlink OFDM transmission. Knowing the channel characteristics of all the users at the base station, the subcarrier allocation algorithm assigns subcarriers to the users in a way that the total transmit power is minimized. The proposed subcarrier and power allocation algorithm offers a low complexity and better performance than the other existing algorithms.

The paper is organized as follows. Next section describes the system model and define the parameters that are going to be used throughout the paper. In Section 3, the proposed algorithm is explained and in Section 4 the performance obtained from simulation results is presented and the complexity is analyzed. Finally some conclusions are drawn.

\section{System Description}

We consider an OFDM system with $K$ users where the total available bandwidth is divided in $N$ orthogonal subcarriers and each transmitter can use the number of subcarriers that are required to satisfy the bit rate needs of its current application. The data are modulated on subcarriers by an IFFT operation. The intersymbol interference can be practically avoided by introducing a guard interval (cyclic prefix between consecutive OFDM symbols. If this guard interval is longer than the maximum delay spread of the channel, the equalizer is very simple at the receiver side.

Let $H_{k, n}$ denotes the value of channel frequency response, $E_{k, n}$ the transmission energy and $b_{k, n}$ the transmission rate for user $k$ on subcarrier $n$.

By taking:

$$
G_{k, n}=\left|H_{k, n}\right|^{2} / \sigma_{k, n}^{2}
$$

We can write:

$$
b_{k, n}=\log _{2}\left(1+\left(E_{k, n} G_{k, n} / \Gamma_{k}\right)\right)
$$

where $\sigma_{k, n}^{2}$ is the variance of the additive white Guassian noise in each subcarrier $n$ for each user $k$, and the gap $\Gamma_{k}$, corresponding to the target Bit Error Rate of user $k$, measures the SNR loss of the actual system with respect to capacity due to the modulation [8].

The goal of the subcarrier and power allocation algorithm is then to fin the best assignment of $b_{k, n}$ so that the overall transmit Energy, the sum of $E_{k, n}$ over all subcarriers and all users, is 
minimized for given transmission rates of the users. This restriction applied to the total energy is motivated by the fact that the base station transmits the signals of all users simultaneously in the downlink.

Let us denote $S_{k} \subset\{1,2, \ldots, N\}$ the set of subcarriers for user $k$. Therefore the problem can be formulated as follows:

$$
\begin{cases}\text { Minimize } & \sum_{n=1}^{N} \sum_{k=1}^{K} E_{k, n} \\ \text { Subject to (s.t.) } & \sum_{n \in S_{k}} b_{k, n} \geq R_{k}, \quad \forall k=1, \ldots, K \\ & S_{i} \cap S_{j}=\phi, \quad \forall i \neq j \\ & S_{1} \cup S_{2} \cup \cdots \cup S_{M} \subset\{1,2, \ldots, N\}\end{cases}
$$

where $R_{k}$ represents the bit rate required by user $k$. The constraint of $S_{i} \cap S_{j}=\phi, \forall i \neq j$ is an FDMA (Frequency Division Multiple Access) restriction in order to ease implementation, i.e. each subcarrier can only be occupied by one user and only one.

\section{Bit Allocation Algorithm}

In order to solve this problem the Energy Minimization Algorithm (EMA) in [9] for single user has been taken as a start and it has been modifie and extended for the multiuser scenario. Let us recall the algorithm in [9]:

Inputs: Channel gains $G_{n}$, total number of subcarriers $N$, BER per user

Outputs: Energy $E_{n}$ and rate $b_{n}$

1. Sort $\mathrm{G}_{n}$ in descending order and set $N^{*}=\mathrm{N}$

2. Calculate:

- $K_{E}=2^{\left[\text {Rate }-\sum_{n=1}^{N *} \log _{2}\left(\frac{G_{n}}{\mathrm{~T}}\right)\right] / N *}$

- $E_{n}^{*}=K_{E}-\frac{\Gamma}{G_{n}}, \quad n=1, \ldots, N^{*}$

3. while $\left(E_{N^{*}}^{*}<0\right), N^{*} \leftarrow N^{*}-1$, go to (2)

4. for $n=1 \ldots N^{*}, b_{n}^{*}=\log _{2}\left(1+E_{n}^{*} G_{n} / \Gamma\right)$

5. for $n=N^{*}+1 \ldots N, E_{n}^{*}=0, b_{n}^{*}=0$

The proposed approach is described as follows:

1. The number of subcarriers allocated to each user is $N_{k}=$ round $(\mathrm{N} / \mathrm{K})$

2. We calculate the quantity $Q_{k}=\sum_{i=1}^{N} G_{k, i}$ for each user.

3. We apply the EMA for each user on starting with the user that has the minimum $Q_{k}$ and we denote $N^{*}=N_{k}$.

The proposed algorithm is a follows:

Inputs: Channel gains $G_{k, n}$, total number of subcarriers $N$, BER per user, bit rate per user $R_{k}$

Outputs: Energy $E_{k, n}$, subcarrier allocation $S_{k}$ and rate $b_{k, n}$

1. The number of subcarriers allocated to each user $N_{k}=$ round $(\mathrm{N} / \mathrm{K})$ 
2. $S_{\text {free }} \leftarrow\{1,2, \ldots, N\}, N_{\text {free }}=\# S_{\text {free }}$

3. Calculate: $Q_{k}=\sum_{i=1}^{N} G_{k, i}$ for $k=1 \ldots, K$

4. Sort users in ascending order such as $Q_{1}<Q_{2}<\cdots<Q_{K}$

5. for each user $k=1: K$

(a) Sort subchannels: $G_{k, S_{\text {free(1) }}}>\cdots>G_{\left.k, S_{\text {free }(\mathrm{free}}\right)}$

(b) Set $N^{*}=N_{k}$

(c) $K_{E}=2^{\left[R_{k}-\sum_{i=1}^{N *} \log _{2}\left(\frac{G_{k, S \text { free(i) }}}{\Gamma}\right)\right] / N^{*}}$

(d) $E_{k, i}=K_{E}-\frac{\Gamma}{G_{\left.k, S_{\text {freed }}\right)}}, i=1, \ldots, N^{*}$

(e) while $\left(E_{k, N^{*}}<0\right), N^{*} \leftarrow N^{*}-1$, go to (c)

(f) $S_{k} \leftarrow S_{\text {free }}\left(1: N^{*}\right), N_{k}=N_{k}^{*}$

(g) $S_{\text {free }} \leftarrow S_{\text {free }}\left(N^{*}+1: N_{\text {free }}\right), N_{\text {free }}=\# S_{\text {free }}$

(h) for $i=1: N^{*}$

- $b_{k, i}=\operatorname{round}\left(\log _{2}\left(1+\frac{E_{k, i} G_{k, S_{k}(i)}}{\Gamma}\right)\right)$

- $E_{k, i}=\left(2^{b_{k, i}-1}\right) \Gamma / G_{k, S_{k}(i)}$

- $\Delta E_{k, i}=E_{k, i}\left(b_{k, i}+1\right)-E_{k, i}\left(b_{k, i}\right)$

(i) end for $\mathrm{i}$

6. end for $j$

Where $\# S_{\text {free }}$ denotes the cardinality of set $S_{\text {free }}$

Given the initial subcarrier allocation, the following algorithm optimizes the bit allocation by using the B-tighten algorithm [10]:

7. $R$ total $_{k}=\sum_{i=1}^{N_{k}} b_{k, i}$

8. for $k=1: K$

(a) while $\operatorname{Rtotal}_{k} \neq R_{k}$

(b) if $\operatorname{Rtotal}_{k}<R_{k}$

- $i \leftarrow \min _{1 \leq n \leq N_{k}} \Delta E_{k, n}$

- $\mathrm{b}_{k, i}=\mathrm{b}_{k, i}+1$

- $E_{k, i}=\left(2^{b_{k, i}-1}\right) \Gamma / G_{k, S_{k}(i)}$

- $\Delta E_{k, i}=E_{k, i}\left(b_{k, i}+1\right)-E_{k, i}\left(b_{k, i}\right)$

(c) else

- $i \leftarrow \max _{1 \leq n \leq N_{k}} \Delta E_{k, n}$

- $b_{k, i}=b_{k, I}-1$

- $E_{k, i}=\left(2^{b_{k, i}-1}\right) \Gamma / G_{k, S_{k}(i)}$

- $\Delta E_{k, i}=E_{k, i}\left(b_{k, i}+1\right)-E_{k, i}\left(b_{k, i}\right)$

(d) end if

(e) end while

9. end for $k$ 


\section{Simulation Results}

In this section, several results obtained by using the proposed algorithm will be presented. Simulation results are divided in two parts. First one analyzes performance and the second one shows the complexity.

\subsection{Performance Analysis}

Without loss of generality, we can assume that the gap $\Gamma_{k}$ is equal for all users, then $\Gamma_{k}=$ $\Gamma$. The following parameters have been used for the simulations: the required BER $P_{e}=$ $10^{-4}$, the power of additive white Gaussian noise is equal to 1 watt, the overall bandwith is $20 \mathrm{MHz}$, the number of subcarriers $N=128$. Two typical WLAN channel models have been used for simulations, namely Hiperlan A and B, A corresponding to a typical offic environment with $50 \mathrm{~ns}$ rms delay spread and B modeling an open NLOS (Non-Line-of-Sight) environment or a large offic with $100 \mathrm{~ns}$ rms delay spread [11]. 100 different and independently generated channels have been simulated and the mean has been taken for results. We evaluate the performance of the proposed algorithm in comparison with BABS-RCG, BABS-ACG algorithms [4], improved BABS-ACG [5] and algorithm in [3].

Figures 1 and 2 show the total transmit power for different number of users and channel models $\mathrm{A}$ and B respectively when $\sum_{k=1}^{K} R_{k}=512$ and $R_{1}=R_{2}=\cdots=R_{K}$. Due to the fact that channel responses are different among users in different locations (multiuser diversity), the total transmit power decreased as the number of users increased.

As shown in Figures 1 and 2 the proposed algorithm performs better than the other algorithms as the number of users increase (number of users higher than 7 for model A and 9 for model B).

From Figures 3 and 4, we observe that the proposed algorithm performs better than all algorithms when $\sum_{k=1}^{K} R_{k}=1024$. This means that the higher the bit rate requirements of the users sharing the communication system the better the proposed algorithm performs with respect to previous ones.

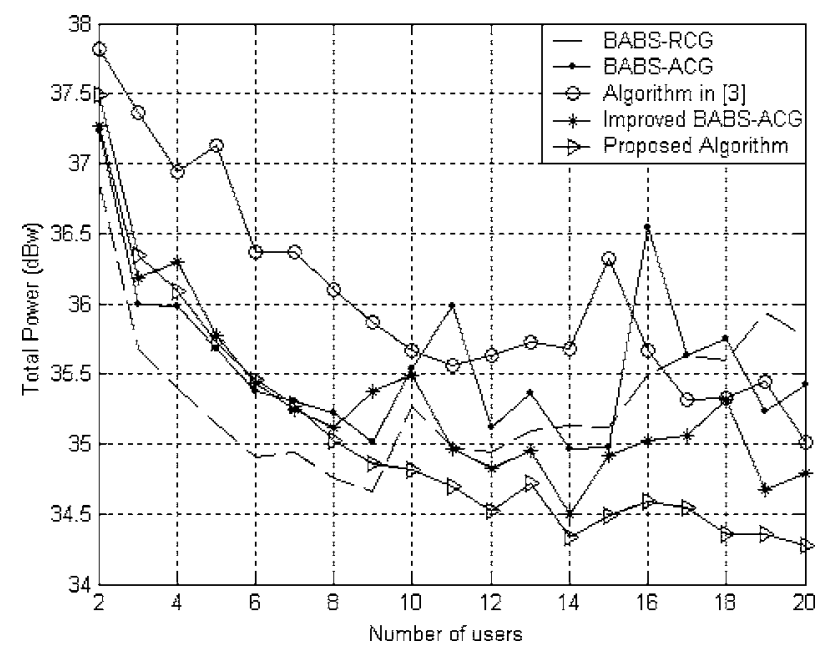

Figure 1. Comparison of the total transmit power for model A when $\sum_{k=1}^{K} R_{k}=512$. 


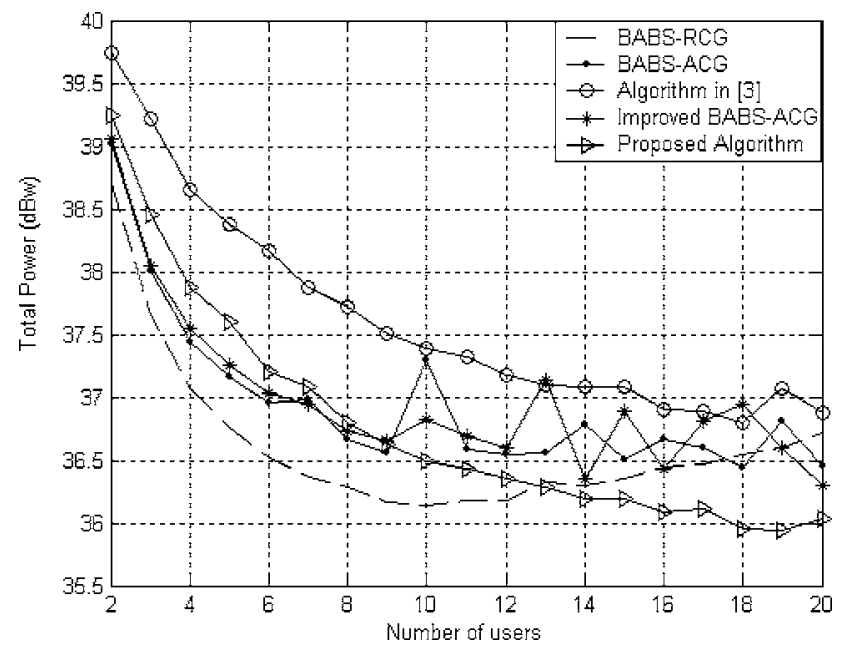

Figure 2. Comparison of the total transmit power for model B when $\sum_{k=1}^{K} R_{k}=512$.

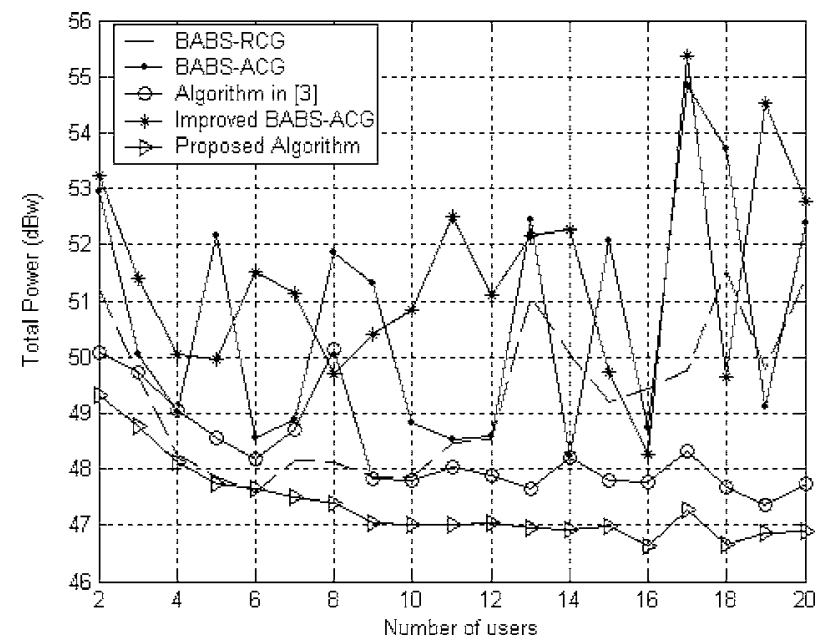

Figure 3. Comparison of the total transmit power for model A when $\sum_{k=1}^{K} R_{k}=1024$.

Moreover, the proposed algorithm is more stable than BABS-RCG, BABS-ACG and Improved BABS-ACG. This is an important characteristic: the entrance of a new user or departure of an existing one must imply a soft change in the situation in order to make the system design tractable.

\subsection{COMPlEXity ANALysis}

The complexity will be measured in terms of the number of iterations needed to implement our algorithm and algorithms introduced above. The comparison is presented in Figure 5. It can be observed that our algorithm needs a lower number of iterations than the others. 


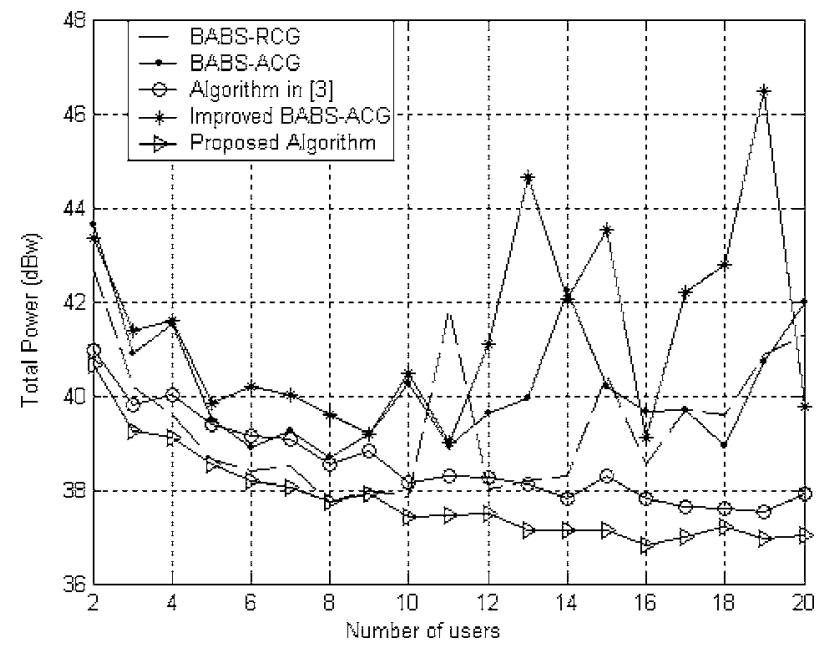

Figure 4. Comparison of the total transmit power for model B when $\sum_{k=1}^{K} R_{k}=1024$.

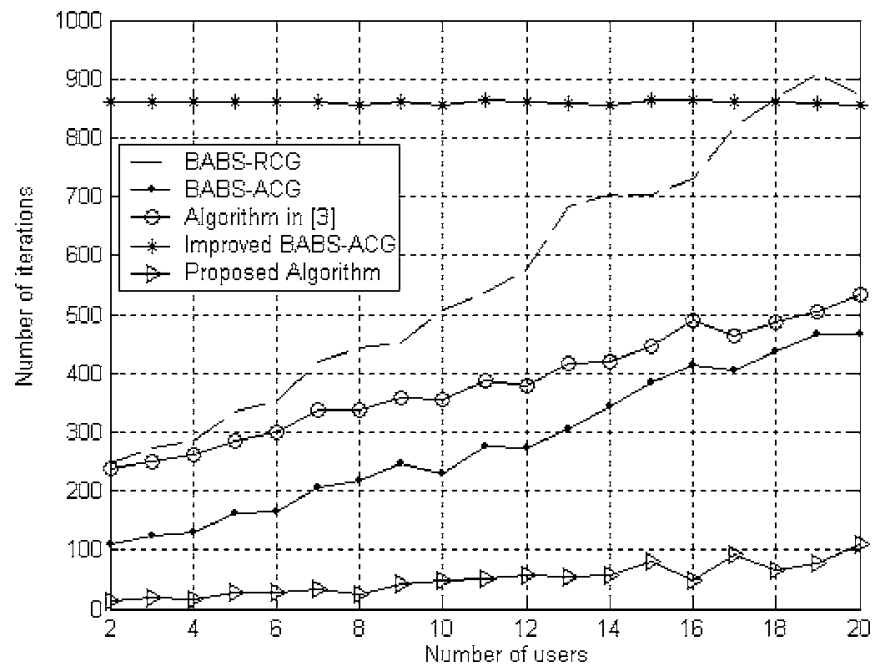

Figure 5. Comparison in number of iterations.

\section{Conclusion}

In this paper, after analyzing the Energy Minimization Algorithm in [9], a new algorithm for subcarrier and power allocation in the downlink of multiuser OFDM transmission has been presented. Simulation results verify that the proposed algorithm performs better than previous ones, with a more noticeable improvement in high bit rate requirements. Moreover, our algorithm is more stable than others, which diverge as the number of users increases. Through the analysis, we have shown that this algorithm has a lower complexity than previous.

\section{Acknowledgment}

This work has been partly funded by the Spanish government with project TIC 2002-03498 (ORISE), Education Chamber of Madrid Community and European Social Fund. 


\section{References}

1. T. Starr, J.M. Cioffi and P.J. Silverman, Understanding Digital Subscriber Line Technology, Englewood Cliffs, NJ: Prentice-Hall, 1999.

2. C.Y. Wong, R.S. Cheng, K.B. Letaief, and R.D. Murch, "Multiuser OFDM with Adaptive Subcarrier, Bit, and Power Allocation", IEEE Journal on Selected Areas in Comm., Vol. 17, pp. 1747-1758, October 1999.

3. I. Kim, H. L. Lee, B. Kim, and Y.H. Lee, "On the Use of Linear Programming for Dynamic Subchannel and Bit Allocation in Multiuser OFDM", in Proc GLOBECOM'01, Vol. 6, pp. 3648-3652, November 2001.

4. D. Kivanc, L. Guoqing, and H. Liu, "Computationally Efficien Bandwidth Allocation and Power Control for OFDMA", IEEE Journal on Selected Areas in Wireless Comm., Vol. 2, pp. 1150-1158, November 2003.

5. L. Zhen, Z. Geqing, W. Weihua, and S. Junde, "Improved Algorithm of Multiuser Dynamic Subcarrier Allocation in 0FDM system", in Proc ICCT, Vol. 2, pp. 1144-1147, April 2003.

6. Z. Shen, J.C. Andrews, and B.L. Evans, "Optimal Power Allocation in Multiuser OFDM Systems", in Proc GLOBECOM'03, Vol. 1, pp. 337-341, December 2003.

7. W. Rhee and J.M. Cioffi "Increasing in Capacity of Multiuser OFDM System Using Dynamic Subchannel Allocation", in Proc VTC, Vol. 2, pp. 1085-1089, May 2000.

8. G.D. Forney and M.V. Eyuboglu, "Combined Equalization and Coding Using Precoding”, IEEE Comm. Magazine, Vol. 29, pp. 25-34, December 1991.

9. L. Hoo, J. Tellado, and J.M. Cioffi "Dual Qos Loading Algorithms for Multicarrier Systems Offering Different CBR Services", in Proc PIMRC, pp. 278-282, September 1998.

10. J. Campello, "Optimal Discrete Bit Loading for Multicarrier Modulation Systems", in Proceedings ISIT, pp. 193, August 1998.

11. "Channel Models for HIPERLAN/2 in Different Indoor Scenarios”, ETSI EP BRAN, 3ERIO85B, March 1998.

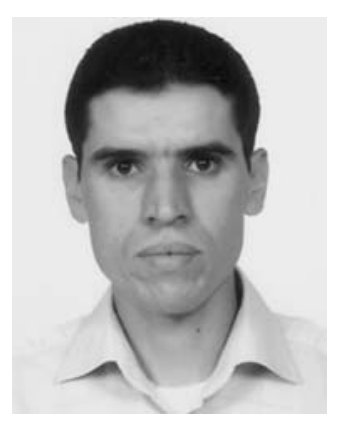

Khalid El Baamrani was born in Ouarzazate, Morocco in 1976. He received the License degree (equiv. B.A.) in electronic engineering from the University of Cadi Ayyad, Marrakech, Morocco, in 1998, the D.E.S.A. (equiv. M.A) in electrical engineering from the University of Cadi Ayyad, Marrakech, Morocco, in 2000, the certificat in engineering of the data-processing networks and telecommunications from the national institute of posts and telecommunications, Rabat, Morocco in 2002 and the Ph.D. degree at University of Cadi Ayyad, Marrakech, Morocco in 2005. His research interests include multicarrier modulation, communication theory, multiuser information theory, OFDM and DSL systems. 


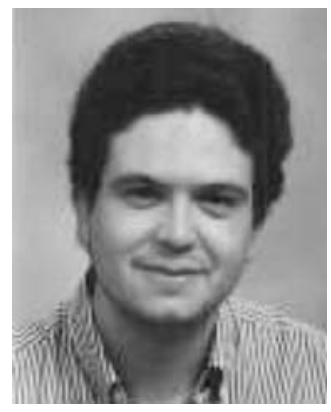

Victor P. Gil Jiménez received the B. Eng. in Telecommunications with honors from University of Alcalá in 1998 and the M. Eng. in Telecommunications and the PhD. degree both from the University Carlos III de Madrid in 2001 and 2005, respectively. He is with the Department of Signal Theory and Communications at the University Carlos III de Madrid as an Assistant Professor. He worked at the Spanish Antarctica Base in 1999 as Communications Staff. He visited University of Leeds and Chalmers Technical University in 2003 and 2004 respectively. His research interests include multicarrier communications and signal processing for wireless systems.

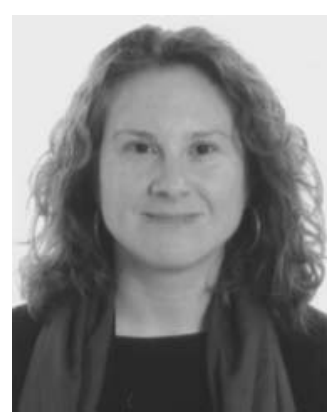

Ana Garcia Armada received the Telecommunication Engineer degree and the Ph.D in Electrical Engineering both from the Polytechnic University of Madrid (Spain) in 1994 and 1998, respectively. She is currently working as an Associate Professor at the University Carlos III de Madrid, where she has occupied several management positions. She has participated in several national and international research projects, most of them related to OFDM. She is coauthor of four books on wireless communications and signal processing. She has published 13 papers in international journals and more than 40 papers in conferences. She has contributed to international organizations such as ITU and ETSI. She has performed research stays in ESA-ESTEC, Kansas University, Stanford University and Bell Labs. Her research interests are simulation of communication systems, multicarrier and MIMO techniques. 